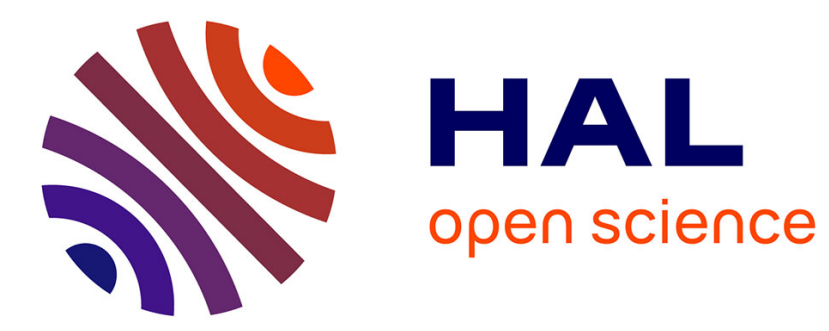

\title{
SANS experiments on micellar nematic mesophases
}

\author{
V. Formoso, Y. Galerne, F. Nicoletta, G. Pepy, N. Picci, R. Bartolino
}

\section{To cite this version:}

V. Formoso, Y. Galerne, F. Nicoletta, G. Pepy, N. Picci, et al.. SANS experiments on micellar nematic mesophases. Journal de Physique IV Proceedings, 1993, 03 (C1), pp.C1-271-C1-277. 10.1051/jp4:1993123 . jpa-00251566

\section{HAL Id: jpa-00251566 https://hal.science/jpa-00251566}

Submitted on 1 Jan 1993

HAL is a multi-disciplinary open access archive for the deposit and dissemination of scientific research documents, whether they are published or not. The documents may come from teaching and research institutions in France or abroad, or from public or private research centers.
L'archive ouverte pluridisciplinaire HAL, est destinée au dépôt et à la diffusion de documents scientifiques de niveau recherche, publiés ou non, émanant des établissements d'enseignement et de recherche français ou étrangers, des laboratoires publics ou privés. 


\title{
SANS experiments on micellar nematic mesophases
}

\author{
V. FORMOSO, Y. GALERNE ${ }^{* * *}$, F.P. NICOLETTA, G. PEPY**, N. PICCI* and R. BARTOLINO \\ Dipartimento di Fisica, Università della Calabria, GNSM and INFM Unità di Cosenza, 87036 Arcavacata \\ di Rende, Cosenza, Italy \\ * Dipartimento di Chimica, Universitd della Calabria, 87036 Arcavacata di Rende, Cosenza, Italy \\ ** Laboratoire Léon Brillouin, CEN-Saclay, 91191 Gif-sur-Yvette cedex, France \\ *** Institut de Physique et Chimie des Matériaux de Strasbourg, Groupe des Matériaux Organiques, I.C.S., \\ 6 rue Boussingault, 67083 Strasbourg cedex, France
}

\begin{abstract}
Lyotropic Nematics are liquid crystalline mixtures characterized by anisotropic aggregates of amphiphilic molecules with finite shape and long range orientational but no positional order.

They are distinguished according to their macroscopic properties in positive and negative uniaxial nematics and in biaxial nematics. Very few notices are available on the structure and on the evolution of the micelles through the different nematic mesophases. We have sndied by Small Angle Neutrons Scattering the potassium laurate, decylammonium hydrochloride, water system which was found to exhibit the three nematic phases as a function of temperature and concentration.

SANS preliminary experiments were performed at PAXY spectrometer of Orphée reactor at Saclay on adequate combinations of tully deuterated components.
\end{abstract}

\section{Introduction}

Lyotropic nematics are anisotropic solutions of amphiphilic molecules packed in finite aggregates, micelles, with long range orientational order. (1)

According to their simmetry properties they are distinguished in positive uniaxial, negative uniaxial and biaxial nematics. It is of ten referred to them respectively as discotic nematics, $\mathrm{Nd}$, cylindrical nematics, $\mathrm{Nc}$ and biaxial nematics. Nbx, from the first idea on the micellar shape of oblate (Nd) and prolate (Nc) ellipsoids.(2) It is possible to pass from discotic micelles to cylindrical ones trough a biaxial mesophase as a function of temperature and/or concentration.

Such richness of mesophases can be obtained only with at least ternary solutions. The absence of biaxial nematics in binary solutions, i.e. acqueous solutions of one amphiphile, suggests that the second amphiphile plays an important role. In particular Charvolin et al. (3) have demonstrated that one component of ternary solution potassium laurate-decanol-water likes to reside in that part of the disc aggregate with lower curvature. The origin of segregation could simply be steric because the different chain length and/or electric because the different polar heads of amphiphiles. According to these experimental data the phase transitions among the different nematics can be due to the disappereance of segregation moving from disc to cylindrical nematics.

Optical analysis and $X$-ray measurements $(4,5)$ have shown that the phase transitions are the result of thermodynamical changes of the axis of overall thuctuations of always biaxial micelles. Small fluctuations of a set of biaxial "bricks" will not change the system biaxiality, but overall fluctuations around an axis will turn the biaxial system into an uniaxial one. In this model the phase transitions are driven by dynamical stability considerations at any temperature.

Nevertheless very few systematic notices are available on the structure of the micelles. In order to understand if the transitions in micellar nematics are mainly governed by changes of the segregation 
processes including in it real variations of micellar shape or by a mechanical stability which keep almost constant the shape, we have studied the potassium laurate-decylammonium hydrochloride-water system by Small Angle Neutron Scattering, SANS. This particular system exhibit all the three nematic phases as a function of temperature and of concentration and it is more stable in time than analogous systems because esterification does not occur. $(6,7)$

We present in this paper the result of some SANS experiments performed on combinations of fully deuterated components (internal contrast). We report the evolution of the micellar dimensions and the aspect anisotropies as a function of temperature.

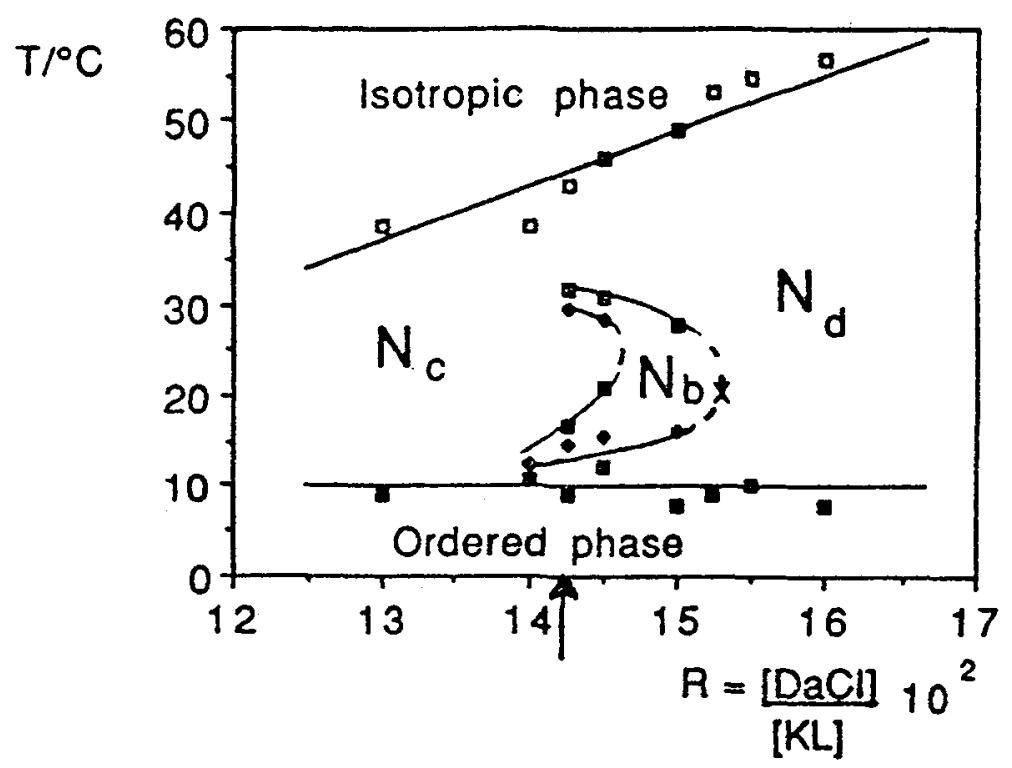

Fig.1) Phase diagram of $\mathrm{Kl}-\mathrm{DaCl}_{2}-\mathrm{D}_{2} \mathrm{O}$ system. The arrow shows the approximative collocation of the samples investigated by SANS.

\section{Experimental}

Fully deuterated compounds were from Cambridge Isotope Laboratories ( D content $>98 \%$, purity > 99\%). Potassium laurate, $\mathrm{KI}$, and decylammonium hydrochloride, $\mathrm{DaCl}$, were prepared as described in a previous work.(7)

Fully deuterated potassium laurate, $\mathrm{Kl}_{\mathrm{d} 23}$, was prepared from fully deuterated lauric acid with the same procedure used for Kl. Decylchloride-d21 was obtained by dissolving fully deuterated decanol in pyridine and adding thyonil chloride in accordance with Gupta et al. (8). Decylammine-d21 was prepared from decylchloride by a Gabriel procedure.

Decylammine was converted in fully deuterated decylammonium hydrochloride, $\mathrm{DaCld} 21$, in accordance with $\mathrm{DaCl}$ preparation (7).

Samples were prepared by weighting the appropiate amounts of the compounds, with $0.05 \mathrm{mg}$ accuracy, in glass tubes. Small quantities of ferrofluids were added in order to improve the alignment in magnetic field. The mixtures were shaken and centrifugated until they were homogeneous between crossed polarizers. The tubes were flame sealed to avoid water losses. 
The mesomorphism presented by the samples as a function of temperature was obtained by a ${ }^{2} \mathrm{H}-\mathrm{NMR}$ technique (7) and by the determination of the optical retardation (5) introduced by the sample when it is

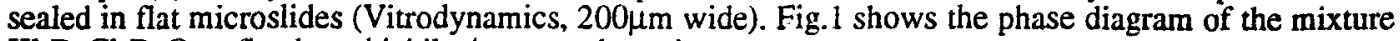
$\mathrm{KL}-\mathrm{DaCl}-\mathrm{D}_{2} \mathrm{O}$ at fixed amphiphiles/water molar ratio,

$$
\frac{[\mathrm{KL}]+[\mathrm{DaCl}]}{\left[\mathrm{D}_{2} \mathrm{O}\right]}=0.05
$$

obtained varying the relative molar ratio of the two amphiphiles

$$
\mathrm{R}=\frac{[\mathrm{DaCl}]}{[\mathrm{KL}]} \quad 0.13<\mathrm{R}<0.17
$$

The phase diagram reports the transition temperatures among the three different nematic phases as a function of relative molar ratio $R$. The biaxial region has an arc shape and it is possible to have a reentrant biaxial and a reentrant disc nematic phase, i.e. the following sequence $\mathrm{Nd}-\rightarrow \mathrm{Nbx} \rightarrow>\mathrm{Nc}->\mathrm{Nbx} \rightarrow \mathrm{Nd}$.

We studied the following samples by SANS: $\mathrm{S} 1\left(\mathrm{Kl}_{\mathrm{d} 23}-\mathrm{DaCl}-\mathrm{D}_{2} \mathrm{O}, \mathrm{R}=0.145\right), \mathrm{S} 2$ (Kl-DaCl-D $2 \mathrm{O}$, $\mathrm{R}=0.142$ ), S3 (Kl-DaCl $\left.\mathrm{d}_{21}-\mathrm{D}_{2} \mathrm{O}, \mathrm{R}=0.150\right)$. Their approximative sollozation in Fig.1 is obtained for $\mathrm{R} \sim 0.142$. The approximation is due to the isotopical exchange which moves, in this case, the phase diagram. SANS measurements were performed with samples sealed in Hellma quartz cells, $1 \mathrm{~mm}$ in thickness, and an horizontal magnetic field of $20 \mathrm{KG}$ was used normal to the neutron beam. The temperature was kept constant at $\pm 0.2^{\circ} \mathrm{C}$. The experiments were performed on the spectrometer PAXY of Orphée reactor at Saclay. The neutron wavelength was $6.00 \AA$ and the scattered neutrons were detected by a $128 \times 128$ elements $2 \mathrm{D}$-multidetector placed at $-2.075 \mathrm{~m}$ far from the samples. Neutron beam was characterized by a diameter $\mathrm{d} \sim 0.7 \mathrm{~cm}$ and by an angular spread $\delta \theta \sim 10^{-2} \mathrm{rad}$. Exposures times were between 900 and 1800 seconds. The investigated q-domain, i.e. the exchanged wavevector range, lies between $0.02 \AA^{-1}$ and $0.16 \AA^{-1}$

\section{Results and discussion}

If we deuterated some components of lyotropic mixture the neutron diffraction patterns will show the proton inhomogeneities inside the sample as schematically represented in Fig.2. As an explicatory example, if we use heavy water, $\mathrm{D}_{2} \mathrm{O}$ and fully deuterated potassium laurate, $\mathrm{Kl}_{\mathrm{d} 23}$, the neutron diffraction pattern will be sensitive to the $\mathrm{DaCl}$ inhomogeneities, i.e. to the segregation of these amphiphilic molecules in the disc phase according to the model of Charvolin et al. (3). The diffraction pattern could not have sufficient contrast in the case of no segregation, i.e. if $\mathrm{DaCl}$ molecules are randomly distributed in the aggregates.

We report in Fig. 3 some 3D images of reconstructed scattered neutron patterns for sample S1, in the disc phase, and S3 in the three different nematic phases. S2 diffraction patterns are similar to those of S3 sample. The magnetic field is along the horizontal axis of the figures. The central part of the diffraction pattern is due to the beam stop. Neutrons scattered by aggregates form some peaks which come out from the noise level. Such peaks reflect the simmetry and order properties of the nematic mesophases. A disc phase shows, in our experimental conditions, a ring (Fig.3.b), a cylindrical phase presents two pronounced peaks along the magnetic field and two lower peaks in the direction perpendicular to $\mathbf{H}$ (Fig.3.d), a biaxial nematic has the same behaviour of a $\mathrm{Nc}$ phase but its peaks have a larger half width (Fig.3.c).

If we assume that the micelles of our lyotropic system have an average occupation volume $V=d_{A} x d_{B} x$ $\mathrm{d}_{\mathrm{C}}\left(\mathrm{d}_{\mathrm{A}}>\mathrm{d}_{\mathrm{B}}>\mathrm{d}_{\mathrm{C}}\right)$, we expect that they will show the largest surface perpendicular to the neutron beam, due to the magnetic field and to the cell walls interactions. In the $\mathrm{Nc}$ phase, the neutron diffraction patterns will be sensitive to $\mathrm{dA}$ along the $\mathrm{H}$ axis and to a convolution of $\mathrm{d}_{\mathrm{B}}$ and $\mathrm{d}_{C}$ dimensions along the direction perpendicular to $\mathrm{H}$ according to the model of tree rotations along the magnetic field.

In the Nd phase the axis of such fiee rotations will be along the neutron beam direction and the diffraction patterns will be characterized by the convolution of $\mathrm{d}_{A}$ and $\mathrm{d}_{B}$ bands in the plane perpendicular to the beam. If the micelles are not well oriented or they feel some tlucruations out firom that plane there will be a contribution of the $\mathrm{d}_{\mathrm{C}}$ band.too. In the $\mathrm{Nbx}$ the rotations are replaced by small amplitude fluctuations and the diffraction is, in principle, due to a convolution of $d_{A}, d_{B}$, and $d_{C}$.

In Fig.3.a the scattered neutron diffaction pattern for $S I$ shows a very poor contrast. The failure of this investigation can be due either to not enough exposure time or to the absence (or not well defined) 

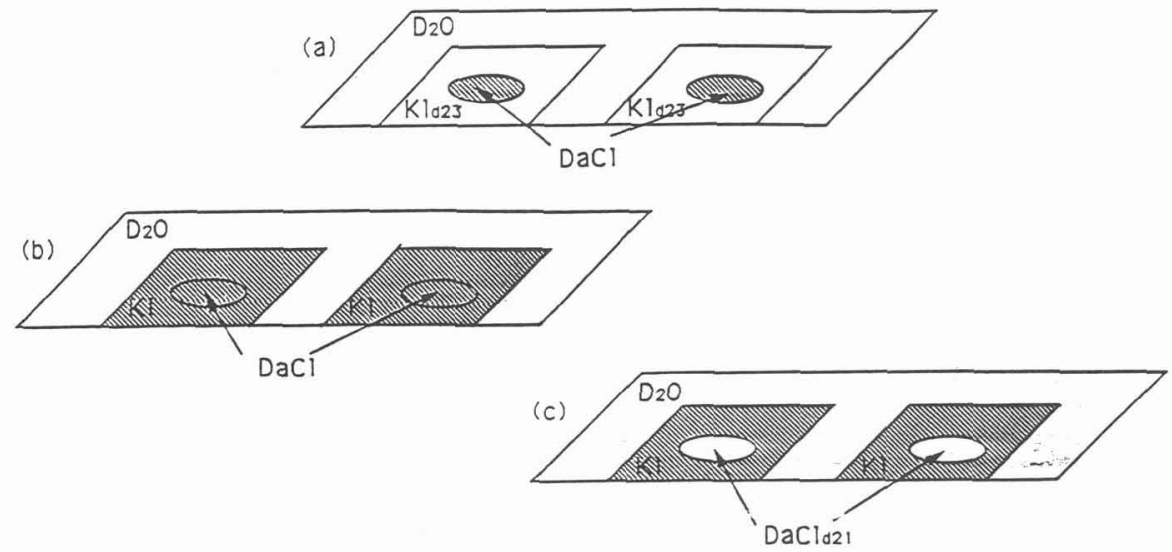

Fig.2) Neutron scatterers, deuterons, are represented by dots in samples S1 (a), S2 (b) and S3 (c). These naive 2D-pictures of lyotropic mixtures should hold in the hypothesis of $\mathrm{DaCl}$ segregation in the lower curvature parts of the micelles.
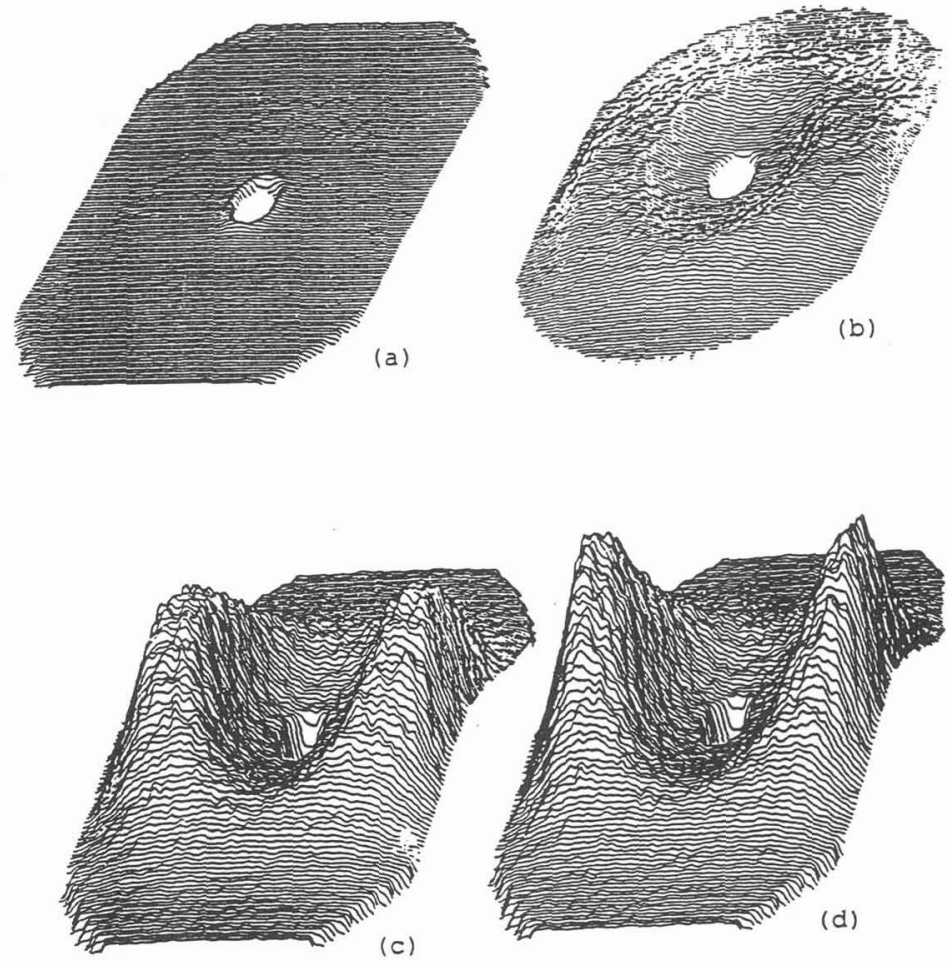

Fig.3) Reconstructed scattered neutron patterns for the disc phase of sample S1 (a) and for the three different nematics of sample $S 3$ (b,c,d are respectively referred to Nd, Nbx, Nc phases). 
segregation of $\mathrm{DaCl}$ molecules inside the micelles. We recall that $\mathrm{DaCl}$ is the amphiphilic component with lower concentration $([\mathrm{DaCl}]:[\mathrm{Kl}] \sim 1: 7)$.

If we assume the micelles as "bricks", the dimensions of which are called $A, B$ and $C(A>B>C)$, it is possible to obtain their values from fits of adequate cuts on the densitometric maps. Each peak on these maps is placed at a certain position in the reciprocal space, $q_{i}(i=A, B, C)$, which gives the related average dimension $d_{i}=2 \pi / q_{i}$ in the direct or real space. The calculated $d i$ are not the $A, B$ and $C$ dimensions of the "bricks" but they take in account the water molecules bound to the micelles, i.e. $d_{i}$ define the average occupational volume of the micelles. Obviously the difference between $d_{i}$ and the real micelles sizes is just the thickness of bound water molecules. As $C$ is theoretically two amphiphilic molecular lengths, i.e. $\sim 26$ $\AA$ and $d_{c}$ is experimentally found to be $-54.0 \pm 0.5 \AA$ for sample $S 2$, we can argue that the bound water is $-14 \AA$ wide. We report the behaviour of $A, B$ and $C$ of samples $S 2$ and $S 3$ as a function of temperature in Fig.4 and Fig.5.

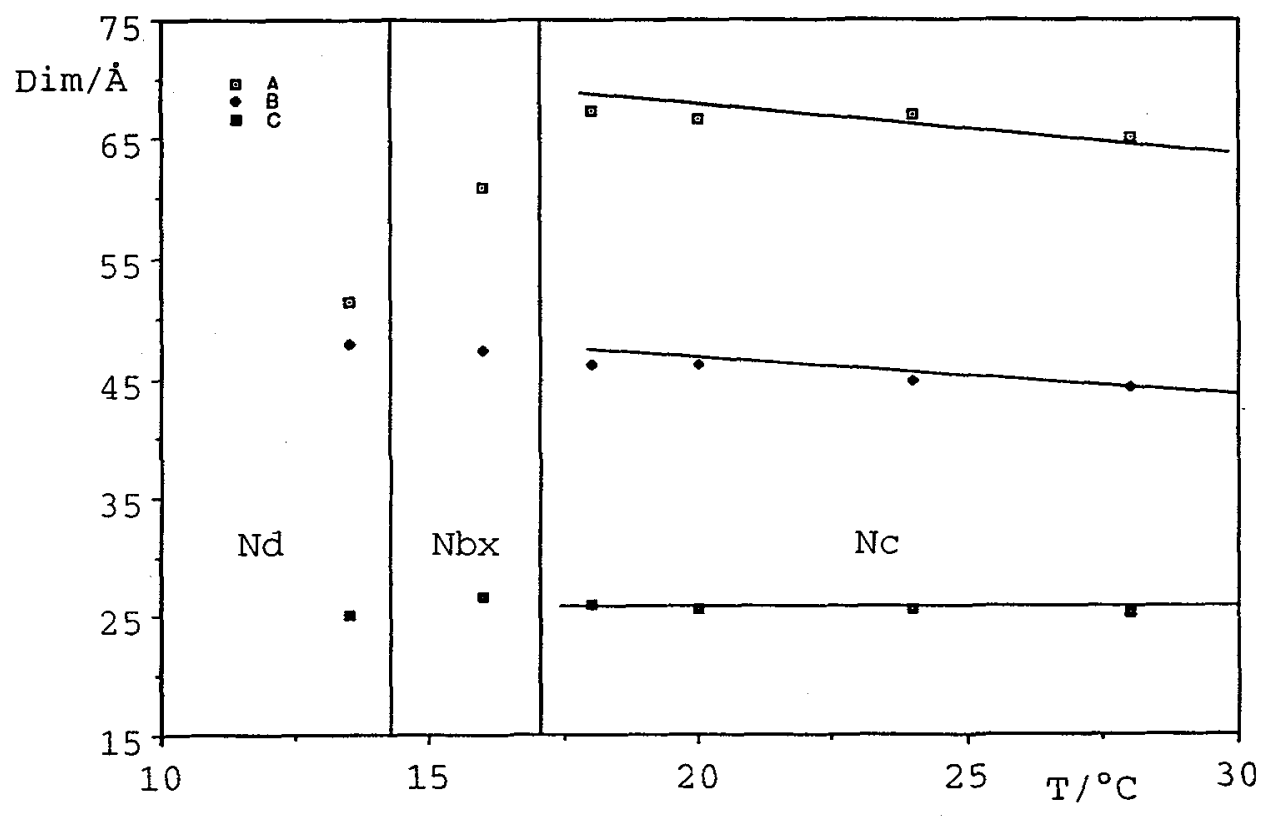

Fig.4) Behaviour of A, B and C micellar dimensions in sample $\mathrm{S} 2$ as a function of temperature.

In Fig. 4 it is possible to underline that:

1) the A dimension increase of $\sim 30 \%$ passing from $51 \pm 1 \AA$ to $67 \pm 1 \AA$ at the transitions $\mathrm{Nd} \rightarrow \mathrm{Nbx} \rightarrow>$ $\mathrm{Nc}$, which confirms that the phase transitions need a continous change of the micellar dimension $A$;

2) such dimensions and consequently the average volume of the micelles are in agreement with X-Ray measurements $(4,5)$;

3) the straight lines seem to suggest a decrease of A and B dimensions in Nc phase as a function of temperature.

Looking at the left part of Fig. 5 we observe the same trend of sample S2 for A, B and C dimensions. A increase of about $20 \%$ and $C$ has almost constant value $\left(\mathrm{d}_{\mathrm{c}} \sim 48.0 \pm 0.5 \AA\right.$, i.e. the bound water is $\sim 11 \AA$ ). $B$ decrease in the $\mathrm{Nbx}$ and $\mathrm{Nc}$ mesophases from $49 \pm 1 \ddot{A}$ to $35.0 \pm 0.5 \AA$ with a slope greater than that of S2. The decrease of A dimension is bigger when it goes down into the discotic reentrant mesophase and, then. into the isotrooic phase. Such results confirm the sugaestions obtained from S2. 
If we calculate the anisotropy ratios, $\mathrm{A} / \mathrm{C}$ and $\mathrm{B} / \mathrm{C}$ (Fig.6), we notice an increase of the first one and a decrease of the second in order to induce a biaxial and a cylindrical mesophase.

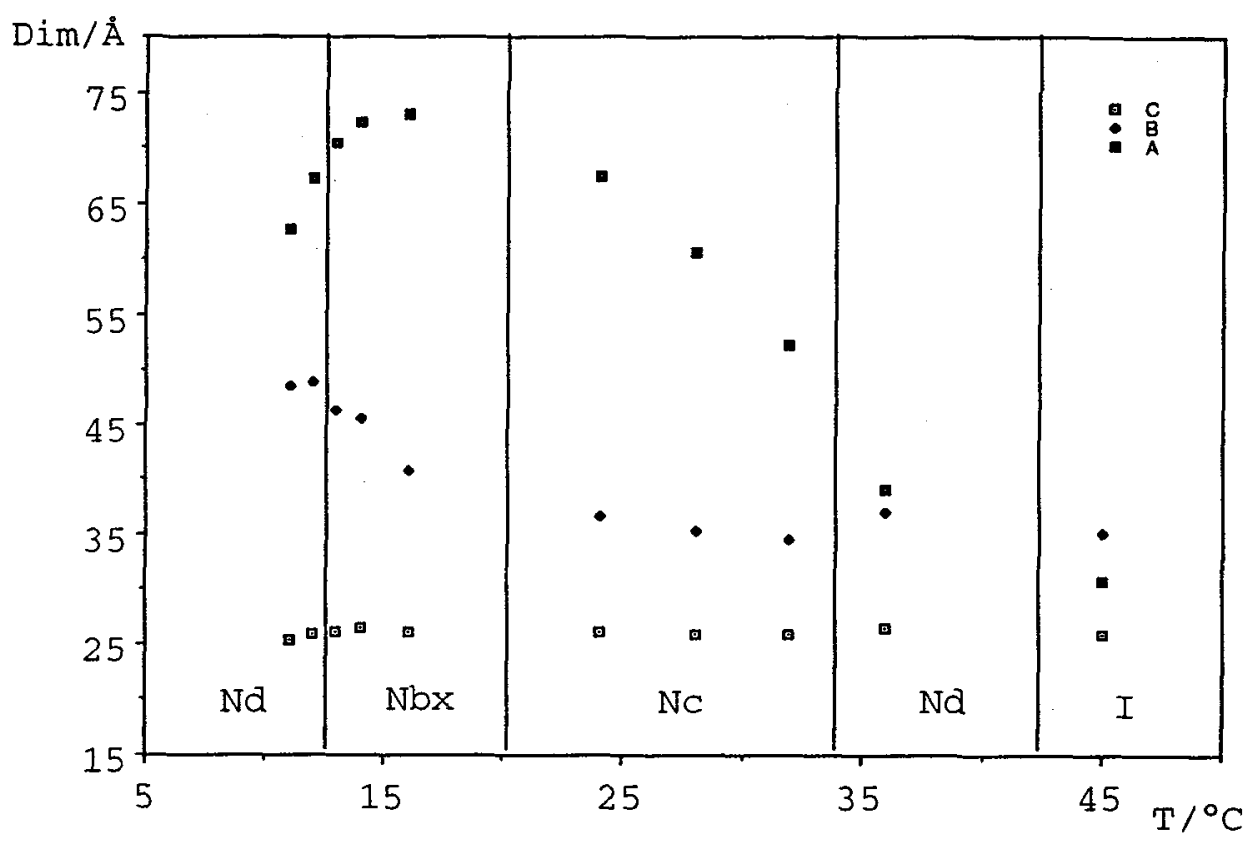

Fig.5) Behaviour of A, B and C micellar dimensions in sample S3 as a function of temperature.

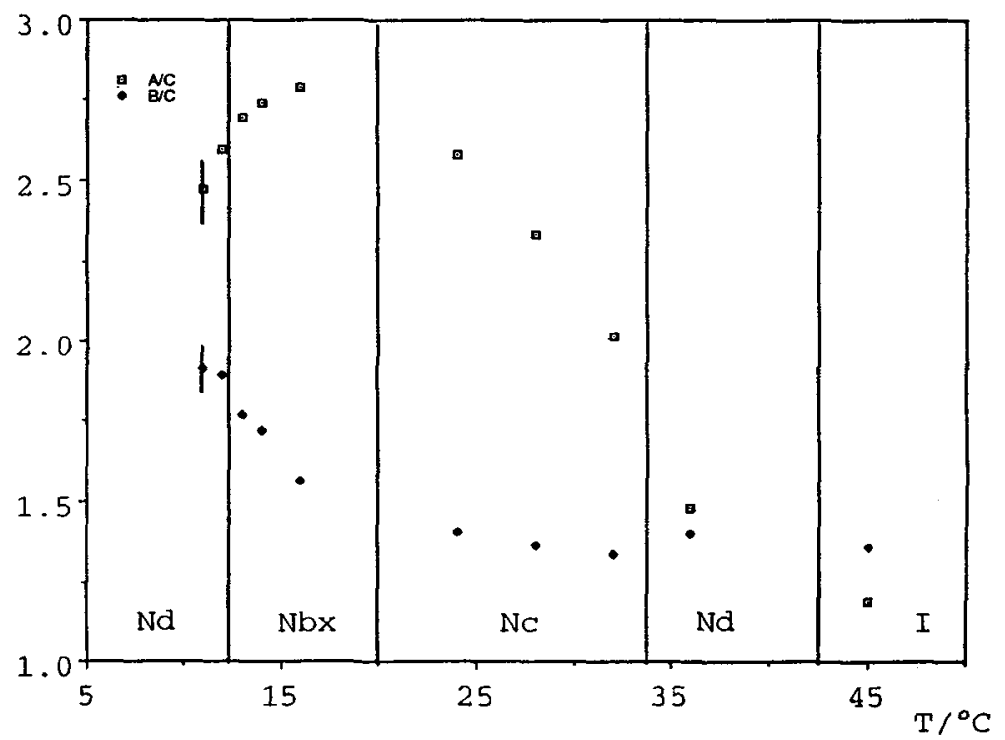

Fig.6) Anisotropy ratios, $\mathrm{A} / \mathrm{C}$ and $\mathrm{B} / \mathrm{C}$, for the micelles of sample $\mathrm{S} 3$ as a function of temperature. 
Conclusions

We made preliminary studies on potassium laurate-decylammonium hydrochloride-water system by Small Angle Neutron Scattering. We adopt an internal contrast technique by using fully deuterated amphiphiles. The experimental dimensions of the micelles are almost the same for different samples and the difference between the aspect ratios is strongly increased in order to obtain the biaxial mesophase.

No definitive informations can be extract from our data, up to now, about the role played by the segregation of $\mathrm{DaCl}$ inside the micelles. Further experiments, which involve two magnetic fields to improve the alignment of the micelles and samples with ligth water to achieve a better contrast, are in progress. Our goal is to quantify better the micellar shape changes and to put in evidence segregational effects. Such effects have been invoked to explain ${ }^{2} \mathrm{H}-\mathrm{NMR}$ measurements performed on the deuterium of selectively deuterated amphiphiles. (9)

\section{References}

1) Lawson K.D. and Flautt T.J., J.Am.Chem.Soc. 89 (1967) 5489.

Radley K., Reeves L.W. and Tracey A.S., J.Phys.Chem. 80 (1976) 174.

Yu L.J. and Saupe A., Phys.Rev.Lett.45 (1980) 1000.

2) Charvolin J., Samulsky E.T. and Levelut A.M., J.de Physique Lett. 40 (1979) L587.

Hendrikx Y. and Charvolin J., J.de Physique 42 (1981) 1427.

Hendrikx Y., Charvolin J., Rawiso M., Liébert L. and Holmes M.C., J.Phys.Chem. 87 (1983) 3391.

Holmes M.C. and Charvolin J., J.Phys.Chem. 88 (1984) 810.

3) Hendrikx Y., Charvolin J. and Rawiso M., Phys.Rev. B33 (1986) 3534.

4) Figueiredo Neto A.M., Galerne Y., Levelut A.M. and Liébert L., J.de Physique Lett. 46 (1985) L499.

Galerne Y., Figueiredo Neto A.M. and Liébert L., J.Chem.Phys. 87 (1987) 1851.

5) Figueiredo Neto A.M., Galerne Y., Levelut A.M. and Liébert L., Liq.Crystals 10 (1991) 751.

6) Oliveira E.A., Liébert L. and Figueiredo. Neto A.M., Liq.Crystals 5 (1989) 1669.

7) Nicoletta F.P., Chidichimo G., Golemme A. and Picci N., Liq.Crystals 10 (1991) 665.

8) Gupta S.K., Rice D.M. and Griftïn R.G., J.Lip.Res. 23 (1982) 197.

9) Nicoletta F.P., Chidichimo G., Golemme A. and Picci N.,"2H-NMR investigation on amphiphilic molecules of nematic lyomesophases" to be published. 